
ARTIFICIAL SATELLITES, Vol. 49, No. 1 - 2014
DOI: 10.2478/arsa-2014-0001

\title{
STUDY ON THE INFLUENCE OF STOCHASTIC PROPERTIES OF CORRECTION TERMS ON THE RELIABILITY OF INSTANTANEOUS NETWORK RTK
}

\author{
Dominik Próchniewicz \\ Warsaw University of Technology \\ Department of Geodesy and Geodetic Astronomy \\ pl. Politechniki 1, 00-661 Warsaw, Poland \\ e-mail: d.prochniewicz@gik.pw.edu.pl
}

\begin{abstract}
The reliability of precision GNSS positioning primarily depends on correct carrier-phase ambiguity resolution. An optimal estimation and correct validation of ambiguities necessitates a proper definition of mathematical positioning model. Of particular importance in the model definition is the taking into account of the atmospheric errors (ionospheric and tropospheric refraction) as well as orbital errors. The use of the network of reference stations in kinematic positioning, known as Network-based Real-Time Kinematic (Network RTK) solution, facilitates the modeling of such errors and their incorporation, in the form of correction terms, into the functional description of positioning model. Lowered accuracy of corrections, especially during atmospheric disturbances, results in the occurrence of unaccounted biases, the so-called residual errors. The taking into account of such errors in Network RTK positioning model is possible by incorporating the accuracy characteristics of the correction terms into the stochastic model of observations. In this paper we investigate the impact of the expansion of the stochastic model to include correction term variances on the reliability of the model solution. In particular the results of instantaneous solution that only utilizes a single epoch of GPS observations, is analyzed. Such a solution mode due to the low number of degrees of freedom is very sensitive to an inappropriate mathematical model definition. Thus the high level of the solution reliability is very difficult to achieve. Numerical tests performed for a test network located in mountain area during ionospheric disturbances allows to verify the described method for the poor measurement conditions. The results of the ambiguity resolution as well as the rover positioning accuracy shows that the proposed method of stochastic modeling can increase the reliability of instantaneous Network RTK performance.
\end{abstract}

Keywords: Network RTK, stochastic model of GNSS observations, instantaneous ambiguity resolution 


\section{INTRODUCTION}

The real-time kinematic positioning method using observations as well as corrections provided by a Ground-Based Augmentation System (GBAS), known as the Network RTK technique, is presently the most commonly utilized method of precision GNSS positioning (Rizos, 2002; Lachapelle and Alves, 2002; Rizos and Han, 2003). Use of a GBAS consisting of a network of reference stations makes possible the determination of ionospheric and geometric (i.e. tropospheric and orbital) network corrections, the so-called correction terms, that guarantee the effective mitigation of spatially correlated errors of GNSS observation. Determining correction terms necessitates the centralized preprocessing of data from the reference stations, know as the network solution. Moreover, in addition to errors mitigation, a very important benefit stemming from the network solution is the possibility of additional control over data from the reference stations with respect to: (i) the quality of GNSS observations, (ii) the stability of the reference frame, and (iii) the accuracy of defined corrections. This results in the Network RTK method being currently the most accurate and reliable method for relative GNSS positioning in real-time.

The reliability of a rover position determined on the basis of carrier-phase GNSS observations may be defined as the resultant of two parameters: solution availability and accuracy. Solution availability describes the possibility of achieving the correct carrierphase ambiguity resolution - i.e. a correct integer estimation of those parameters as well as their acceptance in the validation test. It is only when the ambiguity estimation can be considered valid that the rover solution is a precision solution, known as fixed baseline solution. If not, the ambiguities are assumed as a real-valued and the positioning model solution, the so-called float solution, is an uncertain solution. Such solution type in precise geodetic applications is rejected as being unacceptable and making the rover solution unavailable. This definition of solution availability differs from the accepted definition of this parameter understood in the context of availability of navigation system defined as the ability of the system to provide usable service within the specified coverage area (Booz-Allen and Hamilton, 1996). Solution availability primarily reflects the correctness of the positioning model definition, while system availability is a function of both the physical parameters of the signal propagation center and technical capabilities of transmitter facilities (Hein, 2000). Thus, solution availability is only possible in the case of system availability. As a quantitative parameter, solution availability may be defined on the basis of solution results as the ratio of the number of solutions for which correct ambiguity resolutions were obtained to the total number of solutions for the given period or as a parameter describing the potential probability of correct fixing of ambiguities for the given solution (epoch) - i.e. Ambiguity Resolution Success Rate (Teunissen, 1998) or Probability of Correct Fix (O'Keefe et al., 2006). The solution accuracy describes the accuracy of the rover position estimated for fixed baseline solution and its value can by determined on the basis of position variance estimations or the standard deviation for the given set of solutions.

High level availability and accuracy of Network RTK performance can only be achieved in case of appropriately high strength of GNSS model, which depends on the resultant of the following parameters (Verhagen et al., 2012): satellite geometry, number of observation epochs, number of frequencies, levels of measurement noise, baseline length, and the uncertainty of the applied ionospheric and geometric corrections. Decreasing the number of observed epochs, especially in single system solutions, results in a weakening of model strength, making correct solutions very difficult to achieve or even making them unavailable. 
It is the instantaneous positioning mode that is particularly sensitive in this respect as it only uses single epoch observations. In this mode the solution for every epoch is performed as an independent solution, both for ambiguity resolution and position estimation. Such an approach is particularly desirable in real time applications in light of the minimizing of data acquisition time, which in the case of a kinematic solution is a prerequisite (Odijk, 2001). Additional benefits from instantaneous solution stem from the insensitivity of this method to carrier phase cycle-slips. On the other hand, the small number of degrees of freedom of the instantaneous model results that the ability to detect the model error is limited. This is particularly visible during disturbances in the measurement wave propagation center e.g. ionospheric disturbances or weather fronts. These disturbances can degrade the accuracy of the determined correction terms, resulting in the occurrence of observation biases that are not taken into account by the positioning model, the so-called residual errors. The proper incorporation of such errors in the mathematical model is prerequisite to the optimum estimation of variables.

Existing methods of taking accounting for the residual errors in the stochastic positioning model cannot be fully utilized in the case of an instantaneous solution. These methods may be subdivided into three groups: (i) methods utilizing a priori assumed values of ionospheric and tropospheric residual error in the form of weighed parameters or stochastic constraints (Odijk, 2001; Musa et al., 2003, 2004; Grejner-Brzezinska et al., 2004; Wielgosz et al., 2005); (ii) methods for estimating their values based on the residuals of observations from previous epochs (Han, 1997; Wang et al., 2005); (iii) methods taking advantage of monitoring stations for estimating residual error values (Odijk, 2000). The assumption of a priori values of residual errors in method (i) does not allow to capture the instantaneous current state of the atmosphere, which is critically important in real time applications. The correct estimation of residual errors in the case of method (ii) requires multi-epoch solutions. In effect, it puts limits on the main advantages of instantaneous positioning by sensitizing it to signal loss-of-lock and causing a delay needed for initialization or reinitialization. Utilization of reference stations as stations monitoring the precision of ionospheric and geometric corrections (iii) facilitates the estimation of residual errors that are current for the given epoch, which predisposes this method for instantaneous solutions. However, the exclusion of the monitoring stations from the network solution results in an increase in distances between reference stations used for determining network corrections (Chen et al., 2003; Wanninger, 2004). An additional fault is the need to use the set of monitoring stations in order to find the relation between residual errors and baseline length (Odijk, 2000). In effect, such a solution may prove problematic from the point of view of both correction estimation accuracy and economic issues.

In this paper a different approach to account for the residual errors within the stochastic model is presented. This approach based on the assumption that residual errors of observations remaining after applying network corrections can be described using the variances of those corrections. As a results, it is possible to utilize correction terms variances in the stochastic modeling of the observations. The determination of the correction variances directly in the network solution together with the corrections allows to capture the current residual error values on the basis of observations from single epoch. This predisposes such an approach for application in instantaneous Network RTK positioning. The successive sections of this paper present the proposed stochastic model, called the Network-Based Stochastic Model (NBSM). Specifically, a way for defining a variance-covariance matrix of observations for this model (Sect. 2) as well as its application (Sect. 3) will be described in detail. The results of application of the Network-Based 
Stochastic Model to instantaneous Network RTK positioning, presented in Sect. 4, allow to describe the influence of the accuracy characteristics of the correction terms on the reliability of model solution. The last part of this paper (Sect. 5) contains a summary of presented analyses.

\section{NETWORK RTK POSITIONING MODEL}

The functional model describes the deterministic relationship between observations and variables. It may be assumed that for instantaneous Network RTK positioning, the optimal from the point of view of reliability of parameter estimates is the position model (the Geometry-Based Model) utilizing double-differenced (DD) original phase and code observations (not their linear combinations), where ionospheric and tropospheric delays are introduced a priori as known values in the form of network corrections (Odijk et al., 2000; Odijk, 2008; Takasu and Yasuda, 2010). The linearized observation equation for this model is given by:

$$
\mathbf{y}=\boldsymbol{\rho}_{0}+\mathrm{Ax}+\mathrm{BN}+\mathbf{D}_{\delta} \delta \mathbf{y}+\mathbf{e}
$$

where $\mathbf{y}$ is the vector of DD phase and code measurements, $\boldsymbol{\rho}_{0}$ is the vector of computed for approximate user position DD satellite-receiver ranges, $\mathbf{x}$ is the vector of unknown rover position increments and $\mathbf{N}$ the vector of unknown integer-valued DD ambiguity parameters, $\mathbf{A}$ and $\mathbf{B}$ are design matrices for position parameters of rover and ambiguities respectively and $\mathbf{e}$ is vector of observation errors. Vector $\boldsymbol{\delta} \mathbf{y}$ contains DD ionospheric and geometric correction terms and $\mathbf{D}_{\delta}$ is design matrix to model inter-frequency dependence of correction terms. Denoting observed-minus-computed vector as $\mathbf{l}$, in the form of:

$$
\mathbf{l}=\mathbf{y}-\rho_{0}-\mathrm{D}_{\delta} \delta \mathbf{y}
$$

the functional description of positioning model is presented by the following formula:

$$
\mathrm{E}\{\mathbf{l}\}=\mathbf{A} \mathbf{x}+\mathbf{B N}=\mathbf{l}-\mathbf{e}, \quad \mathbf{x} \in \mathbb{R}^{u}, \quad \mathbf{N} \in \mathbb{Z}^{n}
$$

where $\mathrm{E}\{\cdot\}$ denotes the expectation operator and $u$ and $n$ are the numbers of position parameters and DD ambiguities.

The stochastic model, which describes the stochastic properties of observations, supplements the functional model. Incorporating residual error characteristics into the stochastic description of observations in the form of correction variance, results in the expansion of the variance-covariance observation matrix to include a correction error matrix. The schematic description of the Network-Based Stochastic Model is presented by the following equation:

$$
\mathrm{D}\{\mathbf{l}\}=\mathbf{C}_{l}=\mathbf{C}_{\varepsilon}+\mathbf{C}_{\delta}
$$

where $\mathbf{C}_{l}$ is the variance-covariance matrix of observations, the variance-covariance matrix $\mathbf{C}_{\varepsilon}$ describes the observations noise characteristic and the variance-covariance matrix $\mathbf{C}_{\delta}$ the stochastic properties of correction terms. $\mathrm{D}\{\cdot\}$ denotes the dispersion operator.

Depending on assumptions, the characteristics of measurement noise should take into account the impact on observation variance and covariance of such factors as: elevation dependency, cross-correlation, and time correlation (Tiberius et al., 1999). However, the independence of each solutions in instantaneous mode results in that the time correlation may be neglected (Han, 1997). The methods for stochastic modeling the remaining components of the variance-covariance matrix of observation noise which were applied in the numerical tests are presented in Sect. 3. 
Application the double-differenced observations in the mathematical model results in that the stochastic model of such observations should take into account the differentiation of the observation. Assuming the matrix $\mathbf{C}_{0}$ describes the noise characteristic of the undifferenced observations from a single receiver and $\mathbf{C}_{\Delta}$ the noise characteristic of the single-differenced observations, its relevant variance-covariance matrix for doubledifferenced observations takes (for identical precision characteristics and similar elevation angles for the given satellite for both receivers), according to the propagation law of variances, on the form of (Takasu and Yasuda, 2010):

$$
\mathbf{C}_{\varepsilon}=\mathbf{D}_{\Delta} \mathbf{C}_{\Delta} \mathbf{D}_{\Delta}^{T}=2 \mathbf{D}_{\Delta} \mathbf{C}_{0} \mathbf{D}_{\Delta}^{T}
$$

where $\mathbf{D}_{\Delta}$ is the single-differencing block diagonal matrix for all observations (containing single-differencing matrices $\mathbf{D}$ for the given type of observation).

Determining variance of corrections based on the network solution results in the variance estimation being performed independently for ionospheric and geometric corrections for each observation (for each satellite or for each double-difference). As to the modeling of DD ionospheric refraction for $L 1$ frequency and geometric (non-dispersive) errors (Dai et al., 2003), variance estimations for the master reference station-rover station baseline may be grouped in the following matrix:

$$
\begin{aligned}
& \mathbf{C}_{\delta y}=\left[\begin{array}{ll}
\mathbf{C}_{\delta I} & \\
& \mathbf{C}_{\delta G}
\end{array}\right] \\
& \mathbf{C}_{\delta I}=\operatorname{diag}\left(\begin{array}{llll}
\left(\hat{\sigma}_{\delta I}^{1 j}\right)^{2} & \left(\hat{\sigma}_{\delta I}^{2 j}\right)^{2} & \cdots & \left(\hat{\sigma}_{\delta I}^{k j}\right)^{2}
\end{array}\right) \\
& \mathbf{C}_{\delta G}=\operatorname{diag}\left(\begin{array}{llll}
\left(\hat{\sigma}_{\delta G}^{1 j}\right)^{2} & \left(\hat{\sigma}_{\delta G}^{2 j}\right)^{2} & \cdots & \left(\hat{\sigma}_{\delta G}^{k j}\right)^{2}
\end{array}\right)
\end{aligned}
$$

where $\hat{\sigma}^{2}$ is estimation of a correction variance, subscripts $\delta I$ and $\delta G$ denote ionospheric and geometric biases and superscripts $j$ and $1,2, \ldots, k$ denote the reference satellite and other satellites, respectively. The correction variance matrix of all observations is expressed as follows:

$$
\mathbf{C}_{\delta}=\mathbf{D}_{\delta} \mathbf{C}_{\delta y} \mathbf{D}_{\delta}^{T}
$$

The detailed form of the variance-covariance matrix of observations in the NetworkBased Stochastic Model for dual-frequency phase $\left(L_{1}, L_{2}\right)$ and code $\left(P_{1}, P_{2}\right)$ GNSS observations that takes into account cross-correlation between frequencies $f_{1}$ and $f_{2}$ of the given type of observation (as found in the $\mathbf{C}_{L_{1} L_{2}}$ and $\mathbf{C}_{P_{1} P_{2}}$ matrices) is presented, according to the formula (4), by the following equation:

$$
\begin{aligned}
& \mathrm{D}\{\mathbf{l}\}=\left[\begin{array}{rrr}
2 \mathbf{D C}_{L_{1}} \mathbf{D}^{T}+\mathbf{C}_{\delta I}+\mathbf{C}_{\delta G} & 2 \mathbf{D C}_{L_{1} L_{2}} \mathbf{D}^{T}+\frac{f_{1}^{2}}{f_{2}^{2}} \mathbf{C}_{\delta I}+\mathbf{C}_{\delta G} & \cdots \\
2 \mathbf{D C}_{L_{1} L_{2}} \mathbf{D}^{T}+\frac{f_{1}^{2}}{f_{2}^{2}} \mathbf{C}_{\delta I}+\mathbf{C}_{\delta G} & 2 \mathbf{D C}_{L_{2}} \mathbf{D}^{T}+\frac{f_{1}^{4}}{f_{2}^{4}} \mathbf{C}_{\delta I}+\mathbf{C}_{\delta G} & \cdots \\
-\mathbf{C}_{\delta I}+\mathbf{C}_{\delta G} & -\frac{f_{1}^{2}}{f_{2}^{2}} \mathbf{C}_{\delta I}+\mathbf{C}_{\delta G} & \cdots \\
-\frac{f_{1}^{2}}{f_{2}^{2}} \mathbf{C}_{\delta I}+\mathbf{C}_{\delta G} & -\frac{f_{1}^{4}}{f_{2}^{4}} \mathbf{C}_{\delta I}+\mathbf{C}_{\delta G} & \cdots
\end{array}\right. \\
& \ldots-\quad-\mathrm{C}_{\delta I}+\mathrm{C}_{\delta G} \\
& \cdots \quad-\frac{f_{1}^{2}}{f_{2}^{2}} \mathbf{C}_{\delta I}+\mathbf{C}_{\delta G} \\
& \ldots \quad 2 \mathbf{D C}_{P_{1}} \mathbf{D}^{T}+\mathbf{C}_{\delta I}+\mathbf{C}_{\delta G} \\
& \cdots 2 \mathbf{D C}_{P_{1} P_{2}} \mathbf{D}^{T}+\frac{f_{1}^{2}}{f_{2}^{2}} \mathbf{C}_{\delta I}+\mathbf{C}_{\delta G} \\
& \left.\begin{array}{r}
-\frac{f_{1}^{2}}{f_{2}^{2}} \mathbf{C}_{\delta I}+\mathbf{C}_{\delta G} \\
-\frac{f_{1}^{4}}{f_{2}^{4}} \mathbf{C}_{\delta I}+\mathbf{C}_{\delta G} \\
2 \mathbf{D C}_{P_{1} P_{2}} \mathbf{D}^{T}+\frac{f_{1}^{2}}{f_{2}^{2}} \mathbf{C}_{\delta I}+\mathbf{C}_{\delta G} \\
2 \mathbf{D C}_{P_{2}} \mathbf{D}^{T}+\frac{f_{1}^{4}}{f_{2}^{4}} \mathbf{C}_{\delta I}+\mathbf{C}_{\delta G}
\end{array}\right]
\end{aligned}
$$


It should be additionally stressed that the proposed Network-Based Stochastic Model assumes zero values in the cross-correlation between corrections of the given type for individual observations and between different types of corrections (ionospheric and geometric). This is a simplified approach because the use of linear combinations of double-differenced observations to separate dispersive and non-dispersive errors results in the correlation of individual corrections. However, taking this influence into consideration is coupled with the decomposition of the estimated correction variance from double-differenced to zerodifferenced form. This decomposition requires the acceptance of additional assumptions and may result in additional errors in the stochastic model that are difficult to estimate.

From the point of view of classification of GNSS models, the model described by Eq. (3) and (4) is the Ionosphere-Weighted Troposphere-Weighted Model, while neglecting the correction errors $\left(\mathbf{C}_{\delta}\right)$ in Eq. (4) makes this into the Ionosphere-Fixed TroposphereFixed Model (Teunissen, 1997; Odijk, 2000; Verhagen, 2005). Further on in this paper, a comparative analysis of the solutions to both models for the test data - the Fixed Model and Weighted Model utilizing the Network-Based Stochastic Model - is presented. Differences in the results of the solutions of the compared models allow to estimate the influence of stochastic properties of correction terms on the reliability of instantaneous Network RTK positioning.

\section{APPLICATION PARAMETERS AND SOLUTION ALGORITHM}

A key role in the implementation of the Network-Based Stochastic Model in the given positioning application is played by the choice of the method of correction terms errors estimation. The method is determined by the interpolation model for distance-dependent errors utilized in the network solution. A commonly used spatial interpolation methods for modeling the ionospheric refraction and geometric errors may be divided into two groups (Al-Shaery et al., 2011): (i) the deterministic methods (e.g. Distance-Based Linear Interpolation Method (Gao et al., 1997), Linear Combination Model (Han and Rizos, 1996, 1997), Linear Interpolation Method (Wanninger, 1995), Low-Order Surface Model (Fotopoulos and Cannon, 2001)) and (ii) the geostatistical methods (e.g. Least-Squares Collocation Method (Marel, 1998; Raquet, 1998), Ordinary Kriging Method (Wielgosz et al., 2003; Al-Shaery et al., 2011)). From among these models, only Distance-Based Linear Interpolation Method does not allow the defining of the predicted values errors. The remaining models in the case of a redundancy of reference stations makes possible the estimation of correction variance, but the characteristics of spatial distribution of the variance estimations are varied. Assuming that the network correction errors for the reference station amount to zero and increase with the distance to the nearest station and that their spatial distribution for the area covered by the station network is continuous (Raquet, 1998; Landau et al., 2003, 2007), only geostatistical methods make possible the proper estimation of correction terms variance. On the basis of numerous comparisons of interpolation model applications in the network solution as presented in e.g. (Dai et al., 2004; Geisler, 2006; Próchniewicz, 2011; Al-Shaery et al., 2011), the Ordinary Kriging Method (OKR) may be indicated as the optimal interpolation method for distancedependent errors in regional network, which is characterized as highly precise in determining correction and makes possible the proper estimation of their variance. Numerical tests on positioning model solutions as presented in this paper applied the OKR method, utilizing the gaussian theoretical variogram model. Detailed information relating to the application of the OKR model in network solutions is presented in e.g. (Wielgosz et al., 2003; Al-Shaery et al., 2010, 2011). 
The solving of the instantaneous Network RTK positioning model is performed in three steps (Teunissen, 1993): (i) float solution, (ii) ambiguity resolution, and (iii) fixed baseline solution. An approximate solution is found in the first step (i) by rejecting the integer nature of the ambiguities. The objective function for the float solution is defined by the following equation:

$$
\{\hat{\mathbf{x}}, \hat{\mathbf{N}}\}=\arg \min _{\mathbf{x}, \mathbf{N}}\left\{\|\mathbf{l}-\mathbf{A} \mathbf{x}-\mathbf{B N}\|_{\mathbf{C}_{l}}^{2}, \mathbf{x} \in \mathbb{R}^{u}, \mathbf{N} \in \mathbb{R}^{n}\right\}
$$

with $\|\cdot\|_{\mathbf{C}}^{2}=(\cdot)^{T} \mathbf{C}^{-1}(\cdot)$. The float estimation of the baseline components $(\hat{\mathbf{x}})$ and ambiguities $(\hat{\mathbf{N}})$ is performed using the unconstrained least-square estimation method.

In the successive step (ii), the integer ambiguity estimation is performed on the basis of the float solution (estimated real-valued ambiguities and their variance-covariance matrix $\mathbf{C}_{\hat{N}}$ ). This estimation is performed using the integer least-squares (ILS) method (Teunissen, 1993). The objective function for the ILS method is defined as:

$$
\check{\mathbf{N}}=\arg \min _{\mathbf{N}}\left\{\|\hat{\mathbf{N}}-\mathbf{N}\|_{\mathbf{C}_{\hat{N}}}^{2}, \mathbf{N} \in \mathbb{Z}^{n}\right\}
$$

and its solution is obtained by using a sequential search for ambiguities in the integer search space. This paper applies the ILS estimation implementation in the form of the MLAMBDA method (Chang et al., 2005), which is based on the modified LAMBDA method (Teunissen, 1993, 1995; De Jonge and Tiberius, 1996). This method is the most effective implementation of ambiguity estimation (Hofmann-Wellenhof et al., 2008), especially with respect to kinematic applications (Seeber, 2003).

An important supplement of the ambiguity estimation step is the validation of the integer estimation. This validation on the basis of statistical tests utilizing observation residuals for float and fixed solutions makes possible to verify whether or not the estimated ambiguities may be consider valid (by using the so-called acceptance or integer test) as well as if more that one set of ambiguities pass the acceptance test whether the estimated ambiguities can be considered sufficiently more likely than other for the chosen level of significance $\alpha$ (the so-called discrimination test) (Euler and Schaffrin, 1991; Tiberius et al., 1997). Ambiguity validation for the test data was carried out by using the following discrimination tests: the ratio test (R-ratio, $T_{R}$ ) (Euler and Schaffrin, 1991), the F-ratio test $\left(T_{F}\right)$ (Counselman and Abbot, 1989), and the difference test (D-test, $T_{D}$ ) (Tiberius and De Jonge, 1995). These tests work very well and provide the effective performance of validation, also when they are carried out without the acceptance test (Verhagen, 2004). Definitions of the tests are given by:

$$
\begin{gathered}
T_{R}=\frac{R_{2}}{R_{1}} \geq c_{R} \\
T_{F}=\frac{R_{2}+\hat{\Omega}}{R_{1}+\hat{\Omega}} \geq c_{F} \\
T_{D}=R_{2}-R_{1} \geq c_{D}
\end{gathered}
$$

where $R_{i}$ is the quadratic form of ambiguity residuals for the most likely $(i=1)$ and the second most likely $(i=2)$ integer candidate sets, computed as $R_{i}=\left\|\hat{\mathbf{N}}-\check{\mathbf{N}}_{i}\right\|_{\mathbf{C}_{\hat{N}}}^{2}$ and $\hat{\Omega}$ is the quadratic form of observation residuals for float solution: $\hat{\Omega}=\|\mathbf{l}-\mathbf{A} \hat{\mathbf{x}}-\mathbf{B N}\|_{\mathbf{C}_{l}}^{2}$. The critical values of the tests $\left(c_{R}, c_{F}\right.$ and $\left.c_{D}\right)$ which were applied in the experiment are given in Table 1. 
Tab. 1. Parameters of positioning model application

\begin{tabular}{|c|c|}
\hline Parameter & Settings \\
\hline \multicolumn{2}{|l|}{ Observations } \\
\hline Data & GPS: $L_{1}, L_{2}, P_{1}, P_{2}$ \\
\hline Cut-off angle & \\
\hline Observation model & double difference (maximum elevation) \\
\hline \multicolumn{2}{|l|}{ Functional Model } \\
\hline Model & Geometry-Based \\
\hline Ionospheric delay & network correction \\
\hline Tropospheric delay & a priori model + network correction \\
\hline A priori model of troposphere & Saastamoinen (standard atmosphere) \\
\hline Mapping function & Niell Mapping Function (wet, dry) \\
\hline Satellite ephemeris & Broadcast \\
\hline Receiver antenna model & igs05.atx \\
\hline \multicolumn{2}{|l|}{ Stochastic Model } \\
\hline Model & Network-Based Stochastic Model \\
\hline Carrier-phase variance & $\sigma_{L_{1}}^{s}=0.002+\frac{0.0015}{\sin \epsilon^{s}}[\mathrm{~m}], \sigma_{L_{2}}^{s}=\frac{f_{1}}{f_{2}} \cdot \sigma_{L_{1}}^{s}$ \\
\hline Code variance & $\sigma_{P_{k}}^{s}=100 \cdot \sigma_{L_{k}}^{s}$ \\
\hline Carrier-phase cross-correlation & $\operatorname{cov}_{L_{1} L_{2}}=\frac{f_{1}}{f_{2}} \cdot(0.003)^{2}\left[\mathrm{~m}^{2}\right]$ \\
\hline Code cross-correlation & $\operatorname{cov}_{P_{1} P_{2}}=(0.35)^{2}\left[\mathrm{~m}^{2}\right]$ \\
\hline \multicolumn{2}{|l|}{ Network Solution } \\
\hline Ionospheric correction & Ordinary Kriging \\
\hline Geometric correction & Ordinary Kriging \\
\hline Semivariance model & Gaussian \\
\hline Correction error & variance of OKR prediction \\
\hline \multicolumn{2}{|l|}{ Model Solution } \\
\hline Float solution & least-squares method \\
\hline Ambiguity resolution mode & instantaneous \\
\hline ILS estimation & MLAMBDA \\
\hline \multirow[t]{3}{*}{ ILS validation } & R-ratio, $c_{R}: 2.00,3.00$ \\
\hline & F-ratio, $c_{F}: 1.44,2.00$ \\
\hline & D-test, $c_{R}: 12 \hat{\sigma}_{0}^{2}, 15 \hat{\sigma}_{0}^{2 a}$ \\
\hline
\end{tabular}

${ }^{a} \hat{\sigma}_{0}^{2}$ denotes the a posteriori variance factor for float solution

In the last step (iii) of the solving of the positioning model, the final fix solution is computed by adjusting the unconstrained baseline solutions using the fixed ambiguity estimates. Finally, the position parameters as well as their accuracy characteristic are calculated as (Teunissen, 1995):

$$
\begin{gathered}
\check{\mathbf{x}}=\hat{\mathbf{x}}-\mathbf{C}_{\hat{x} \hat{N}} \mathbf{C}_{\hat{N}}^{-1}(\hat{\mathbf{N}}-\check{\mathbf{N}}) \\
\mathbf{C}_{\check{x}}=\mathbf{C}_{\hat{x}}-\mathbf{C}_{\hat{x} \hat{N}} \mathbf{C}_{\hat{N}}^{-1} \mathbf{C}_{\hat{N} \hat{x}}
\end{gathered}
$$

Table 1 presents the detailed parameters used in the application of models described in Sect. 2 . 


\section{EXPERIMENT ANALYSIS}

In order to define the influence of including accuracy characteristic of the correction terms in the stochastic model of observations on the reliability of instantaneous Network RTK solution results (especially the results of ambiguity estimation and validation as well as position estimation), the Fixed Model and Weighted Model were compared. Both of the compared models are based on the same functional description and only the stochastic descriptions are different, as it was presented in Sect. 2. It should be also noted that only the one difference in computational process of compared models lies in forming the variance-covariance matrix $\mathbf{C}_{l}$ in float solution (9).

The network used in the test is a fragment of Austrian regional network EPOSA (Echtzeit Positionierung Austria, http://www.eposa.at) supplemented with two stations (KIRC and WIND) of the local NetFocus network (Punktgenaues Echtzeit Positionierungsservice NetFocus, http://www.netfocus.at) (Fig. 1). This network is characterized by significant differences in station elevation (up to $550 \mathrm{~m}$ ), which may have an unfavorable impact on the modeling accuracy of tropospheric refraction. Moreover, the used data was collected on March 9, 2012 during a severe ionospheric disturbances - the maximum Kp index reached 80 (Bartels, 1957). This makes it possible to test the proposed solutions subject to difficult measuring conditions, which significantly restrict the availability of instantaneous solution. Two reference stations (WIND and LIEZ) located within the area covered by the reference station network were excluded from the network solution and used as test stations (user stations). The various lengths of the test baselines between the master reference station (WEYE) and the user stations $(29.9 \mathrm{~km}$ and $46.1 \mathrm{~km}$ ) allows to analysis of dependence of solution results on distance to the reference station. In the test 12-hour observation session with an interval of 15 seconds and cut-off angle of $10^{\circ}$ was used.

The ionospheric and geometric corrections were determined using the OKR method. Figures 2 and 3 present the effectiveness of the reduction of ionospheric and geometric

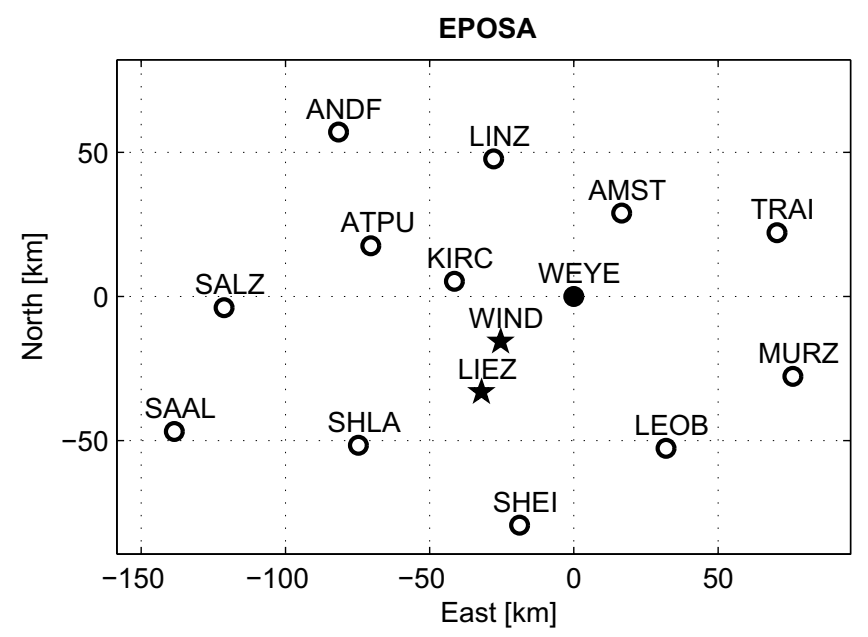

Fig. 1. Map of the reference stations test network: $\circ$ - reference station, $\bullet-$ master reference station, $\star-$ user's stations 

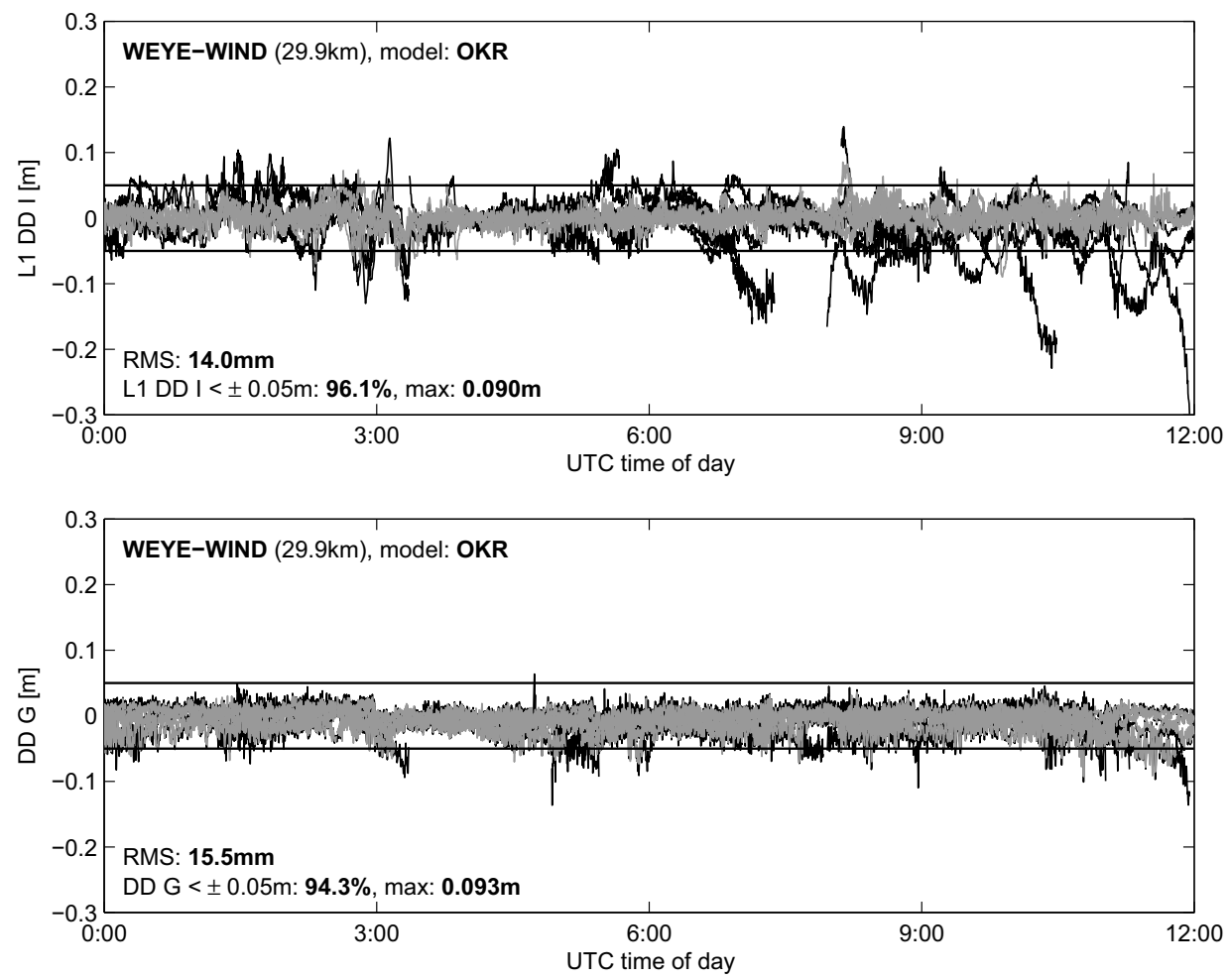

Fig. 2. DD ionospheric delays for $L 1$ (top) and geometric errors (bottom) for the test baseline WEYE-WIND: black lines - actual values, gray lines - residual values following application of network correction terms

components of distance-dependent errors by using network corrections. The color black marks the DD ionospheric refraction (for $L 1$ ) as well as geometric errors (following the elimination of the main part on the basis of the a prior model, see Table 1). The color gray denotes their residual values following application of correction terms. The level of ionospheric errors expressed through the RMS was reduced as a result of the application of corrections of approximately 60-70\%. Also, the number of epochs for which ionospheric errors do not exceed $\pm 5 \mathrm{~cm}$ threshold was increased from $54 \%$ to $96 \%$ for the WEYE-WIND baseline and from $27 \%$ to $92 \%$ for the WEYE-LIEZ. In the case of geometric errors, the reduction in the RMS error amounted to approximately $20 \%$, while the number of epochs for which geometric errors did not exceed $\pm 5 \mathrm{~cm}$ increased by approximately $5-10 \%$. The RMS errors of observation residual errors remaining after the application of corrections are $\pm 15 \mathrm{~mm}$ for both the ionospheric and geometric components of both test baselines, where a small dependency is visible in the level of the error and the length of the baseline for the ionospheric errors.

Ambiguity estimations for the two compared models were performed in instantaneous mode independently for each epoch using the MLAMBDA method. Table 2 shows the results of the comparison of the ambiguity parameter estimations and the reference values calculated on the basis of known coordinates of the user stations. These results were presented in the form of the Ambiguity Estimation Success Rate (AESR) indicator calculated as the ratio of the number of correct solutions to the total number of solutions as well as the number of epochs characterized by the given result. Figures 4 and 5 present the resultant errors of the user position calculated on the basis of the comparison of the estimated position and known positions assumed to be error free. These graphs 

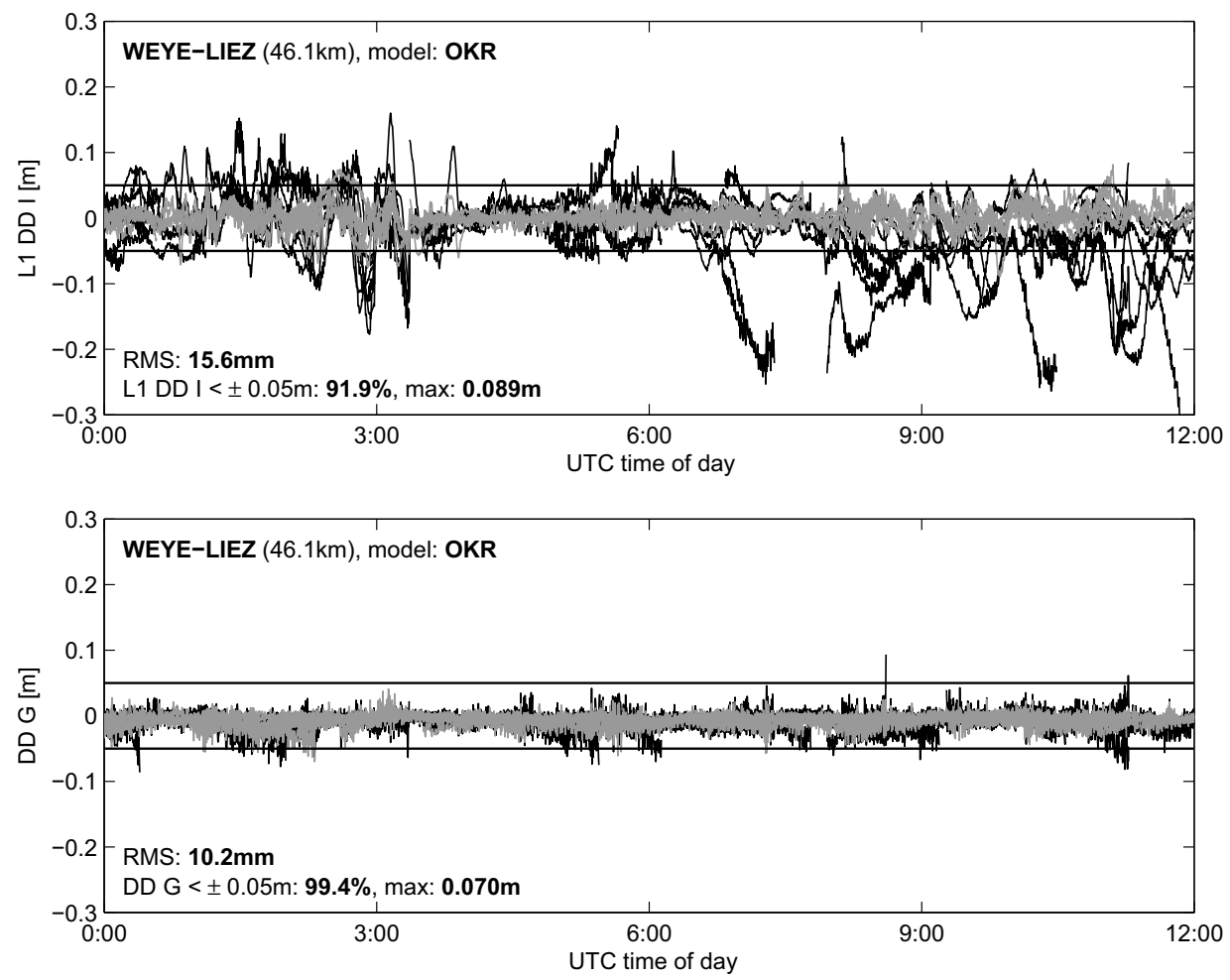

Fig. 3. DD ionospheric delays for $L 1(t o p)$ and geometric errors (bottom) for the test baseline WEYE-LIEZ: black lines - actual values, gray lines - residual values following application of network correction terms

Tab. 2. Ambiguity estimation statistic

\begin{tabular}{|c|c|c|c|c|c|}
\hline \multirow[t]{3}{*}{ Baseline } & \multicolumn{2}{|c|}{ Fixed Model } & \multicolumn{2}{|c|}{ Weighted Model } & \multirow[t]{2}{*}{ Difference } \\
\hline & $\check{\mathbf{N}}=\mathbf{N}$ & $\check{\mathbf{N}} \neq \mathbf{N}$ & $\check{\mathbf{N}}=\mathbf{N}$ & $\check{\mathbf{N}} \neq \mathbf{N}$ & \\
\hline & \multicolumn{5}{|c|}{ [Success Rate $\backslash$ epoch] } \\
\hline WEYE-WIND & $95.3 \%$ & $4.7 \%$ & $99.9 \%$ & $0.1 \%$ & $+4.6 \%$ \\
\hline$(29.9 \mathrm{~km})$ & 2684 & 132 & 2813 & 3 & +129 \\
\hline WEYE-LIEZ & $91.1 \%$ & $8.9 \%$ & $98.9 \%$ & $1.1 \%$ & $+7.8 \%$ \\
\hline$(46.1 \mathrm{~km})$ & 2564 & 250 & 2782 & 32 & +218 \\
\hline
\end{tabular}

are presented in a logarithmic scale, where solutions for which ambiguity was estimated incorrectly are marked by the symbol $\star$. The analysis of Table 2 and Fig. 4 and 5 shows that utilizing the accuracy characteristic of the correction terms in the NBSM increases the number of correct ambiguity estimation solutions of approximately $5-8 \%$ to a level of $98.9-99.9 \%$. What is also particularly important, the incorrect ambiguity estimation for the Fixed Model were obtained for the epoch for which the residual errors exceed \pm 0.05-0.06 m (e.g., prior to 3:00 and after 9:00 UTC) - see Fig. 2 and 3. Correct ambiguity estimations for those epochs using the Weighted Model confirms that the way in which residual errors are taken into account in the stochastic model for instantaneous positioning as proposed in NBSM form is an effective method that increases the reliability of ambiguity estimations. 

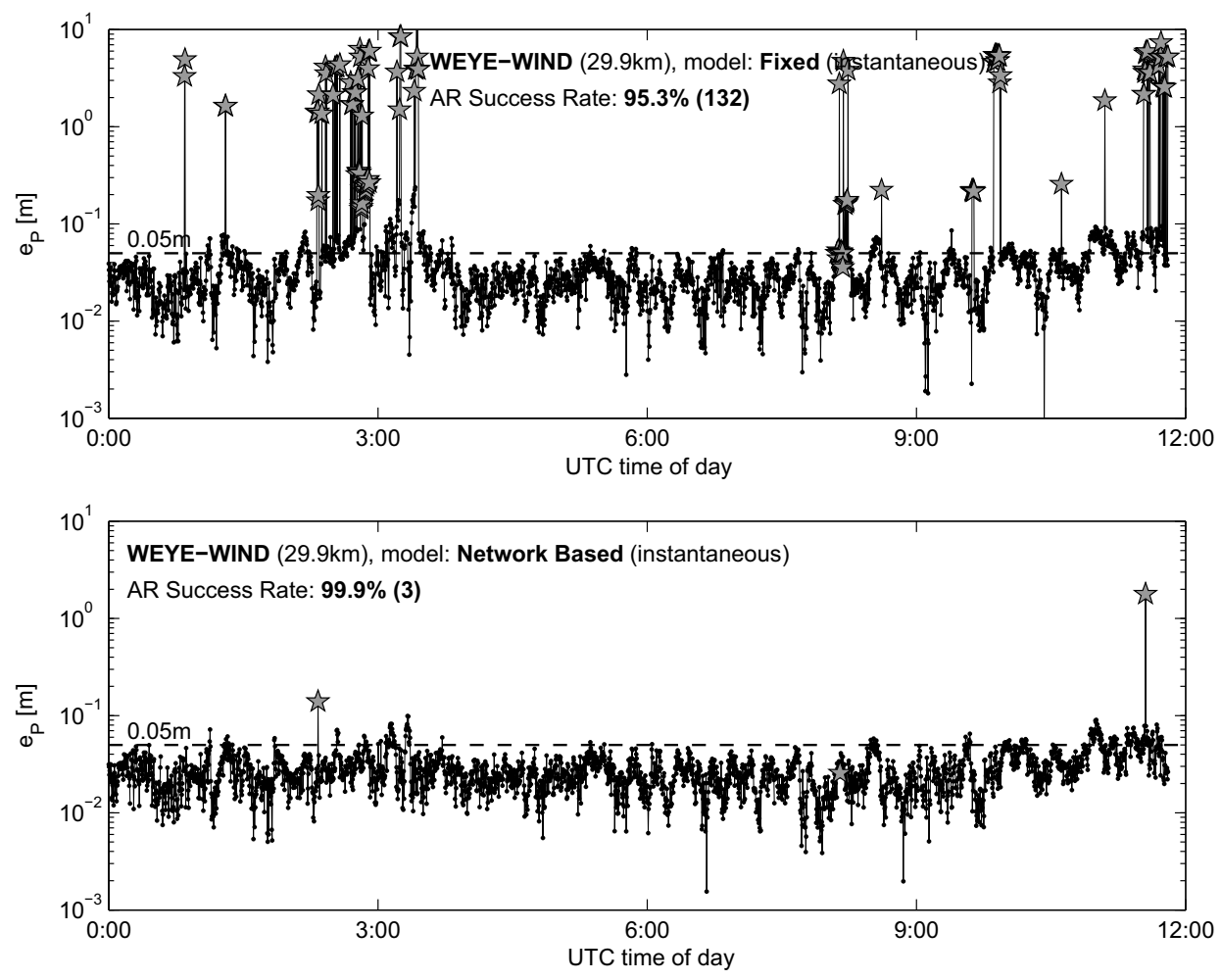

Fig. 4. Position errors of user station for the test baseline WEYE-WIND: Fixed Model (top), Weighted Model (bottom)
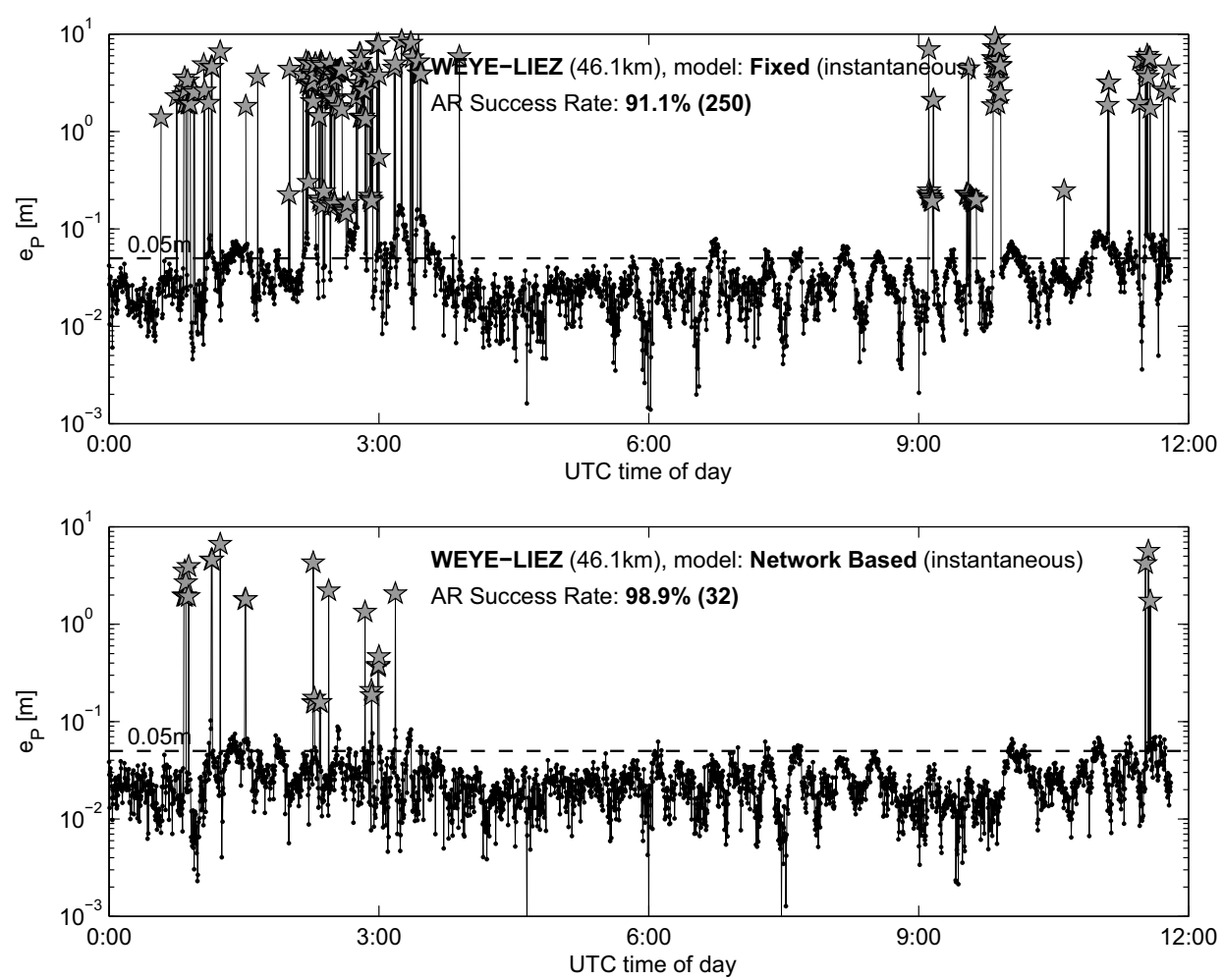

Fig. 5. Position errors of user station for the test baseline WEYE-LIEZ: Fixed Model (top), Weighted Model (bottom) 
Tab. 3. Ambiguity validation statistic

\begin{tabular}{|c|c|c|c|c|}
\hline \multirow[t]{3}{*}{ Baseline } & \multirow[t]{2}{*}{ Model } & \multicolumn{3}{|c|}{ AR Success Rate \False Acceptance } \\
\hline & & \multicolumn{2}{|c|}{$[\% \backslash$ epoch] } & {$[c: \backslash \%]$} \\
\hline & R-ratio $c_{R}$ : & 2.00 & 3.00 & $\min (\beta=0)$ \\
\hline \multirow{2}{*}{$\begin{array}{l}\text { WEYE-WIND } \\
(29.9 \mathrm{~km})\end{array}$} & Fixed & $85.1 \% \backslash 3$ & $75.9 \% \backslash 0$ & 2.34: $81.5 \%$ \\
\hline & Weighted & $97.3 \% \backslash 0$ & $90.3 \% \backslash 0$ & 1.59: $98.8 \%$ \\
\hline \multirow{3}{*}{$\begin{array}{l}\text { WEYE-LIEZ } \\
(46.1 \mathrm{~km})\end{array}$} & Fixed & $76.8 \% \backslash 10$ & $63.9 \% \backslash 0$ & 2.84: \\
\hline & Weighted & $92.3 \% \backslash 0$ & $81.3 \% \backslash 0$ & 1.64: $95.0 \%$ \\
\hline & F-ratio $c_{F}:$ & 1.44 & 2.00 & $\min (\beta=0)$ \\
\hline \multirow{2}{*}{$\begin{array}{l}\text { WEYE-WIND } \\
(29.9 \mathrm{~km})\end{array}$} & Fixed & $90.4 \% \backslash 10$ & $82.9 \% \backslash 3$ & 2.21: $80.6 \%$ \\
\hline & Weighted & $98.3 \% \backslash 0$ & $92.9 \% \backslash 0$ & 1.33: $99.0 \%$ \\
\hline \multirow{3}{*}{$\begin{array}{l}\text { WEYE-LIEZ } \\
(46.1 \mathrm{~km})\end{array}$} & Fixed & $83.9 \% \backslash 32$ & $73.8 \% \backslash 4$ & 2.63: $62.7 \%$ \\
\hline & Weighted & $95.3 \% \backslash 2$ & $81.6 \% \backslash 0$ & 1.54: $93.0 \%$ \\
\hline & D-test $c_{D}$ : & $12 \hat{\sigma}_{0}^{2}$ & $15 \hat{\sigma}_{0}^{2}$ & $\min (\beta=0)$ \\
\hline \multirow{2}{*}{$\begin{array}{l}\text { WEYE-WIND } \\
(29.9 \mathrm{~km})\end{array}$} & Fixed & $93.1 \% \backslash 89$ & $92.5 \% \backslash 80$ & $268 \hat{\sigma}_{0}^{2}: 18.8 \%$ \\
\hline & Weighted & $96.3 \% \backslash 0$ & $94.9 \% \backslash 0$ & $9 \hat{\sigma}_{0}^{2}: 97.9 \%$ \\
\hline \multirow{2}{*}{$\begin{array}{l}\text { WEYE-LIEZ } \\
(46.1 \mathrm{~km})\end{array}$} & Fixed & $88.1 \% \backslash 150$ & $87.2 \% \backslash 128$ & $324 \hat{\sigma}_{0}^{2}: \quad 9.8 \%$ \\
\hline & Weighted & $91.8 \% \backslash 8$ & $88.7 \% \backslash 5$ & $32 \hat{\sigma}_{0}^{2}: 66.3 \%$ \\
\hline
\end{tabular}

Validation of ambiguity estimation for the test data was performed for three discrimination tests that assume various critical values (see Table 1). Validation results are presented in Table 3 in the form of: (i) the Ambiguity Resolution Success Rate (ARSR), which is the ratio of the number of solutions for which ambiguity was estimated correctly and accepted by the discrimination test to the total number of solutions, and (ii) the False Acceptance (FA) indicator presents the number of epochs for which incorrect ambiguity estimations were accepted by the test. Thus, the FA indicator described Type II errors whose probability of occurrence $(\beta)$ defining the power of the test: $1-\beta$. The last column of Table 3 presents the ARSR for a hypothetical critical value of the test selected as a minimal value for which no Type II errors occur. Thus, this indicator defines the greatest possible effectiveness of solutions that can be achieved at $100 \%$ power of the validation test. This allows to compare a potential for solving tested models in conjunction with the validation tests. The analysis of Table 3 shows that using NBSM allows to increase the ARSR indicator in the case of all discrimination tests as used, where the difference amounts to approximately $15 \%$ for the R-ratio, $10 \%$ for the F-ratio, and 3\% for the D-test. Also, the power of the validation tests for ambiguity solutions for the Weighted Model achieves a greater value as compared with the Fixed Model, which makes it possible to assume lower critical values in the test. Hence, differences in the maximum achievable ARSR for $\beta=0$ amount to approximately $18 \%$ for the WEYE-WIND baseline and approximately $30 \%$ for the WEYE-LIEZ for R-ratio and F-ratio. In the case of the D-test, these differences reach approximately 55-80\%. Also visible is a clear dependency between the effectiveness of the ambiguity resolution and the baseline length, but the difference in ARSR for both baselines is clearly smaller in the case of applying NBSM. 
Tab. 4. Statistic of rover position residuals (estimated minus true position components) for correct fixed solution

\begin{tabular}{|c|c|c|c|c|c|c|c|c|}
\hline \multicolumn{2}{|c|}{ Baseline } & \multicolumn{3}{|c|}{ Fixed Model } & \multicolumn{3}{|c|}{ Weighted Model } & \multirow{2}{*}{$\begin{array}{c}\text { RMS } \\
\text { reduction }\end{array}$} \\
\hline & & RMS & $\sigma$ & $\bar{e}$ & RMS & $\sigma$ & $\bar{e}$ & \\
\hline & & \multicolumn{6}{|c|}{$[\mathrm{mm}]$} & [\%] \\
\hline WEYE-WIND & $\mathrm{dN}:$ & 11.5 & 11.4 & 1.5 & 7.9 & 7.8 & 0.8 & $31.3 \%$ \\
\hline \multirow{2}{*}{$(29.9 \mathrm{~km})$} & $\mathrm{dE}:$ & 14.2 & 9.0 & -11.0 & 12.7 & 5.9 & -11.3 & $10.6 \%$ \\
\hline & $\mathrm{dU}:$ & 34.2 & 24.9 & 23.4 & 27.0 & 17.5 & 20.7 & $21.0 \%$ \\
\hline WEYE-LIEZ & $\mathrm{dN}:$ & 13.1 & 13.1 & -0.4 & 8.7 & 8.7 & -1.0 & $33.6 \%$ \\
\hline \multirow[t]{2}{*}{$(46.1 \mathrm{~km})$} & $\mathrm{dE}:$ & 10.6 & 9.7 & 4.2 & 7.1 & 6.0 & 3.8 & $33.0 \%$ \\
\hline & $\mathrm{dU}:$ & 35.4 & 28.0 & 21.7 & 26.1 & 17.7 & 19.2 & $26.3 \%$ \\
\hline
\end{tabular}

In the last of the performed tests, position estimation results were compared for the fixed baseline solution for both models. Figures 6 and 7 present errors in determining position for horizontal components ( $\mathrm{dN}$ and $\mathrm{dE}$, left figure) and for vertical component (dU, right figure) calculated on the basis of a comparison of estimated user station positions and their reference (true) position. The analysis of the precision of the position determination utilized only those solutions for which correct ambiguity estimations were obtained. Table 4 presents RMS, standard deviation and mean of the position residuals for individual components. The last column shows the level of reduction in RMS for individual components received for the solution applying NBSM as compared with the Fixed Model. Analysis of the results lead to the conclusion that use of the NBSM model makes possible a significant improvement in the precision of the estimated positions by approximately $30 \%$ for horizontal components, and approximately $20 \%$ for vertical component. The standard deviation of the estimated positions in the NBSM model does not exceed $\pm 9 \mathrm{~mm}$ for $\mathrm{dN}, \pm 6 \mathrm{~mm}$ for $\mathrm{dE}$, and $\pm 18 \mathrm{~mm}$ for $\mathrm{dU}$, which is an improvement with respect to the Fixed Model of approximately $3 \mathrm{~mm}$ for horizontal components and $8 \mathrm{~mm}$ for vertical component. Most of the estimated positions for NBSM fall within the $\pm 3 \mathrm{~cm}$ range on the horizontal plane the $\pm 5 \mathrm{~cm}$ for the vertical component, which is a very good result for the positioning of the instantaneous Network RTK. However, a clear dependence of the accuracy of the position estimation on length of the baseline as well as a certain bias for elevation components for both baselines and for the dE component for WEYE-WIND baseline can be noted. The reason behind this error may be lowered accuracy in the modeling of geometric errors in mountainous terrain or errors in the application of calibration models for receiver antennas.

\section{CONCLUSIONS}

The paper presents analyses relating to the influence of the stochastic characteristics of network correction terms on the reliability of instantaneous Network RTK performance. Assessments of this influence were defined on the basis of a comparison of two models performance: the Ionosphere-Weighted Troposphere-Weighted Model and the IonosphereFixed Troposphere-Fixed Model. The difference in the definition of the two models stems from the incorporation of ionospheric and geometric correction variances determined 

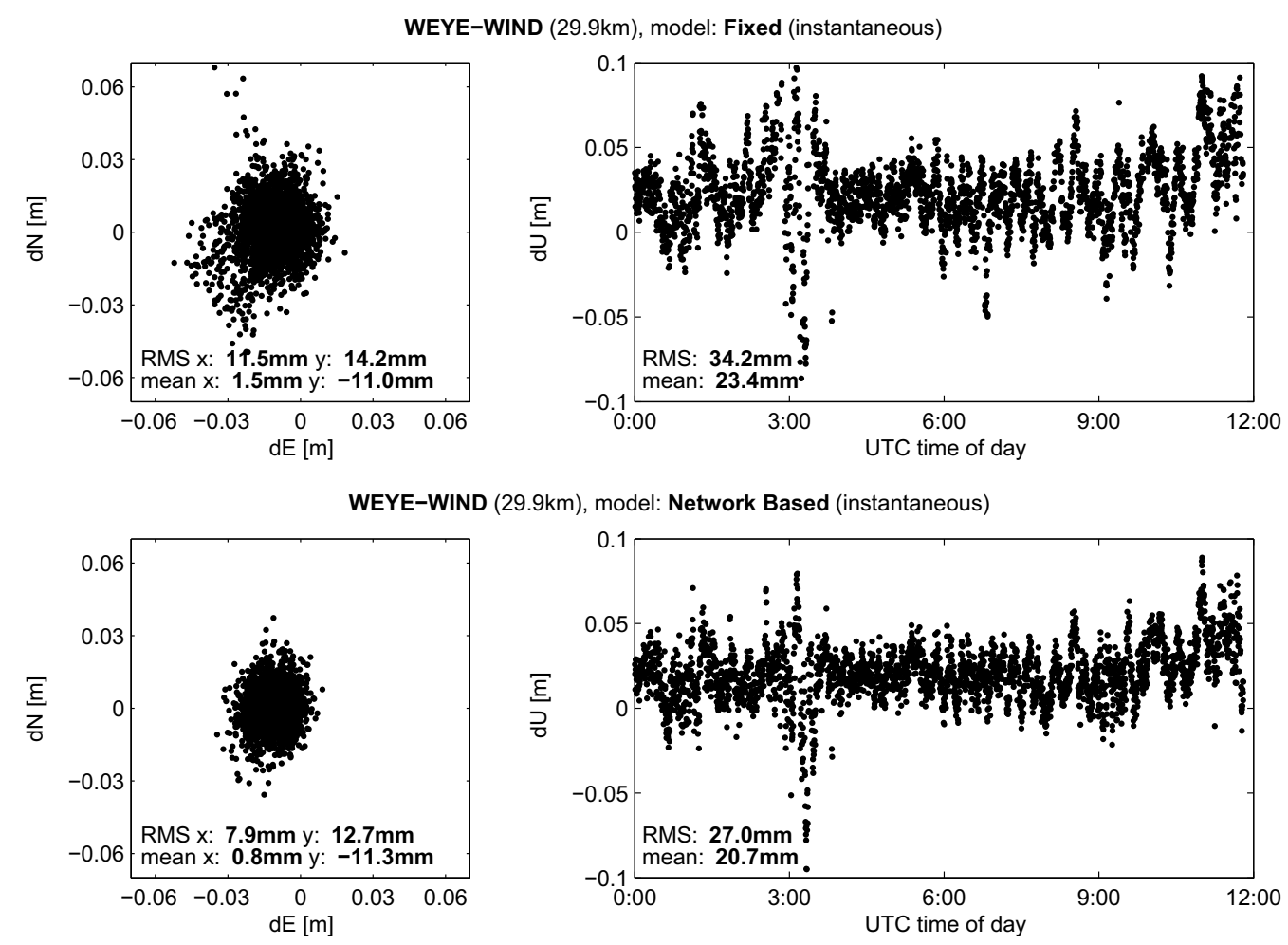

Fig. 6. Horizontal (left) and vertical (right) components of position residuals of user station for the test baseline WEYE-WIND: Fixed Model (top), Weighted Model (bottom)
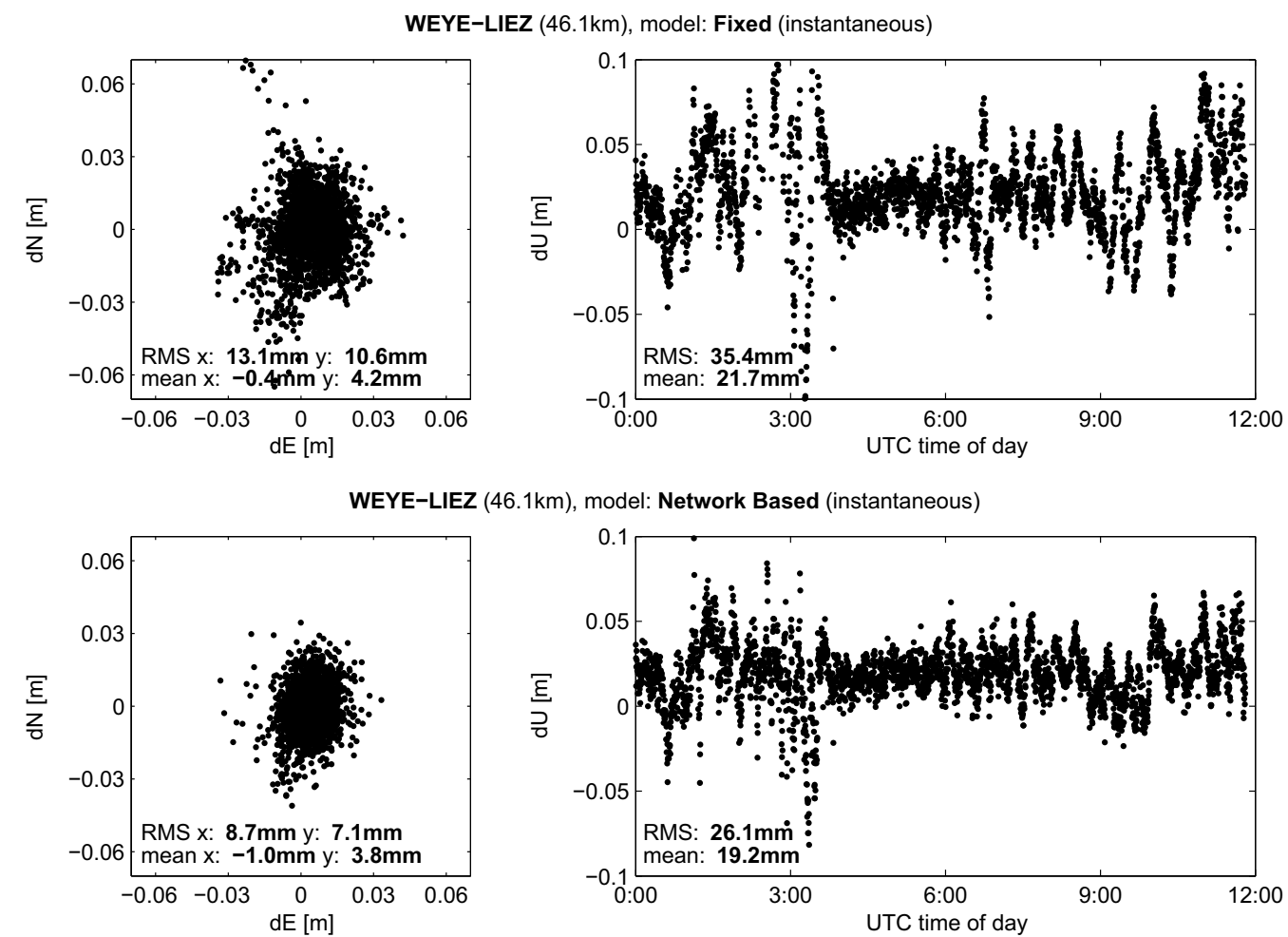

Fig. 7. Horizontal (left) and vertical (right) components of position residuals of user station for the test baseline WEYE-LIEZ: Fixed Model (top), Weighted Model (bottom) 
together with corrections in the network solution into the stochastic model. Such a model, called the Network-Based Stochastic Model, makes possible the taking into account of residual errors in the positioning model. This paper presents a method for applying the proposed model and the results of the model solution. It is on their basis can be stated that the incorporation of correction accuracy characteristics into the Network RTK positioning model allows for increased reliability of solution in instantaneous mode in both the availability and solution accuracy aspects.

Acknowledgements. This work was supported by the grants funded by Polish National Science Centre (DEC-2012/07/N/ST10/03728) and Faculty of Geodesy and Cartography, Warsaw University of Technology. R. Weber and G. Möller from Vienna University of Technology are acknowledged for providing GPS data from EPOSA and NetFocus networks.

\section{REFERENCES}

Al-Shaery A., Lim S., and Rizos C. (2010). Functional models of ordinary kriging for medium range real-time kinematic positioning based on Virtual Reference Station technique. Proceedings of the 23th International Technical Meeting of the Satellite Division of the Institute of Navigation, ION GNSS 2010, 2513-2521.

Al-Shaery A., Lim S., and Rizos C. (2011). Investigation of different interpolation models used in Network-RTK for the Virtual Reference Station technique. Journal of Global Positioning Systems, 10(2), 136-148.

Bartels J. (1957). The technique of scaling indices K and Q of geomagnetic activity. Annales of the International Geophysical Year, 4, 215-226.

Booz-Allen and Hamilton (1996). Second draft for-working group review, proposed European Baseline Radionavigation Plan, appendix b. Technical report, ICAO AllWeather Operations Panel.

Chang X., Yang X., and Zhou T. (2005). MLAMBDA: a modified LAMBDA method for integer least-squares estimation. Journal of Geodesy, 79, 552-565.

Chen X., Landau H., and Vollath U. (2003). New tools for Network RTK integrity monitoring. Proceedings of the 16th International Technical Meeting of the Satellite Division of the Institute of Navigation, ION GPS/GNSS 2003, 1355-1360.

Counselman C.C. and Abbot R. (1989). Method of resolving radio phase ambiguity in satellite orbit determination. Journal of Geophysical Research, 94(B6), 7058-7064.

Dai L., Han S., Wang J., and Rizos C. (2004). Comparison of interpolation algorithms in network-based GPS techniques. Journal of the Institute of Navigation, 50(4), 277-294.

Dai L., Wang J., Rizos C., and Han S. (2003). Predicting atmospheric biases for real-time ambiguity resolution in GPS/GLONASS reference station networks. Journal of Geodesy, 76, 617-628.

De Jonge P.J. and Tiberius C.C.J.M. (1996). The LAMBDA method for integer ambiguity estimation: implementation aspects. Technical report, Delft Geodetic Computing Centre, Delft University of Technology.

Euler H.J. and Schaffrin B. (1991). On a measure for the discernability between different ambiguity solutions in static-kinematic GPS mode. IAG Symposia no. 10\%, Kinematic Systems in Geodesy, Surveying, and Remote Sensing, 285-295.

Fotopoulos G. and Cannon M.E. (2001). An overview of multireference station methods for cm-level positioning. GPS Solutions, 4(3), 1-10. 
Gao Y., Li Z., and McLellan J.F. (1997). Carrier phase based regional area differential GPS for decimeter-level positioning and navigation. Proceedings of the 10th International Technical Meeting of the Satellite Division of the Institute of Navigation, ION GPS 1997, 1305-1313.

Geisler I. (2006). Performance improvement of Network RTK positioning. Proceedings of the 2006 National Technical Meeting of The Institute of Navigation, 869-880.

Grejner-Brzezinska D.A., Wielgosz P., Kashani I., Smith D.A., Spencer P.S.J., Robertson D.S., and Mader G.L. (2004). An analysis of the effects of different network-based ionosphere estimation models on rover positioning accuracy. Journal of Global Positioning Systems, 3(1-2), 115-131.

Han S. (1997). Quality-control issues relating to instantaneous ambiguity resolution for real-time GPS kinematic positioning. Journal of Geodesy, 71, 351-361.

Han S. and Rizos C. (1996). GPS network design and error mitigation for real-time continuous array monitoring systems. Proceedings of the 9th International Technical Meeting of the Satellite Division of the Institute of Navigation, ION GPS 1996, 1827 1836.

Han S. and Rizos C. (1997). Instantaneous ambiguity resolution for medium-range GPS kinematic positioning using multiple reference stations. Proceedings of the International Association of Geodesy Symposia, 118, Advances in Positioning and Reference Frames, 283-288.

Hein G.W. (2000). From GPS and GLONASS via EGNOS to Galileo - positioning and navigation in the third millennium. GPS Solutions, 3(4), 39-47.

Hofmann-Wellenhof B., Lichtenegger H., and Wasle E. (2008). GNSS Global Navigation Satellite Systems: GPS, GLONASS, Galileo \& more. Springer-Verlag, Wien.

Lachapelle G. and Alves P. (2002). Multiple reference station approach: overview and current research. Journal of Global Positioning Systems, 1(2), 133-136.

Landau H., Chen X., Kipka A., and Vollath U. (2007). Latest developments in Network RTK modeling to support GNSS modernization. Journal of Global Positioning Systems, $6(1), 47-55$.

Landau H., Vollath U., and Chen X. (2003). Virtual Reference Stations versus broadcast solutions in Network RTK - advantages and limitations. Proceedings of GNSS 2003 The European Navigation Conference, Graz, Austria.

Marel van der H. (1998). Virtual GPS reference stations in the Netherlands. Proceedings of the 11th International Technical Meeting of the Satellite Division of the Institute of Navigation, ION GPS 1998, 49-58.

Musa T.A., Wang J., and Rizos C. (2004). A stochastic modelling method for network-based GPS positioning. European Navigation Conference, ECN GNSS2004.

Musa T.A., Wang J., Rizos C., and Satirapod C. (2003). Stochastic modelling for networkbased GPS positioning. The 6th International Symposium on Satellite Navigation Technology Including Mobile Positioning and Location Serivces, Melbourne, Australia.

Odijk D. (2000). Weighting ionospheric corrections to improve fast GPS positioning over medium distances. Proceedings of the 13th International Technical Meeting of the Satellite Division of the Institute of Navigation, ION GPS 2000, 1113-1123.

Odijk D. (2001). Instantaneous precise GPS positioning under geomegnetic storm conditions. GPS Solutions, 5(2), 29-42.

Odijk D. (2008). GNSS solutions: mathematical models. Inside GNSS, 3(2), 22-24.

Odijk D., Marel van der H., and Song I. (2000). Precise GPS positioning by applying ionospheric corrections from an active control network. GPS Solutions, 3(3), 49-57. 
O'Keefe K., Petovello M., Lachapelle G., and Cannon M.E. (2006). Assessing probability of correct ambiguity resolution in the presence of time-correlated errors. Navigation: Journal of the Institute of Navigation, 53(4), 269-282.

Próchniewicz D. (2011). A study on mitigation of the distance-dependent biases in the Network RTK technique. Reports on Geodesy, 90(1), 397-407.

Raquet J.F. (1998). Development of a method for kinematic GPS carrier-phase ambiguity resolution using multiple reference receivers. Ph.D. thesis, The University of Calgary.

Rizos C. (2002). Network RTK research and implementation - a geodetic perspective. Journal of Global Positioning Systems, 1(2), 144-150.

Rizos C. and Han S. (2003). Reference station network based RTK systems - concepts and progress. Wuhan University Journal of natural Sciences, 8(2B), 566-574.

Seeber G. (2003). Satellite Geodesy: foundations, methods, and applications. Walter de Gruyter, Berlin, New York, 2nd completely rev. and extended edition.

Takasu T. and Yasuda A. (2010). Kalman-filter-based integer ambiguity resolution strategy for long-baseline RTK with ionosphere and troposphere estimation. Proceedings of the 23rd International Technical Meeting of the Satellite Division of the Institute of Navigation, ION GNSS 2010, 161-171.

Teunissen P.J.G. (1993). Least squares estimation of the integer GPS ambiguities. Invited lecture, Section IV Theory and Methodology, IAG General Meeting, Beijing.

Teunissen P.J.G. (1995). The least-squares ambiguity decorrelation adjustment: a method for fast GPS integer ambiguity estimation. Journal of Geodesy, 70, 65-82.

Teunissen P.J.G. (1997). The geometry-free GPS ambiguity search space with a weighted ionosphere. Journal of Geodesy, 71, 730-383.

Teunissen P.J.G. (1998). Success probability of integer GPS ambiguity rounding and bootstrapping. Journal of Geodesy, 72, 606-612.

Tiberius C.C.J.M. and De Jonge P.J. (1995). Fast positioning using LAMBDA-method. Proceedings of the 4 th International Symposium on Differential Satellite Navigation Systems, DSNS 1995(paper no. 30).

Tiberius C.C.J.M., Jonkman N., and Kenselaar F. (1999). The stochastics of GPS observables. GPS World, 10(2), 49-54.

Tiberius C.C.J.M., Teunissen P.J.G., and De Jonge P.J. (1997). Kinematic GPS: performance and quality control. Int. Symp. on Kinematic Systems in Geodesy, Geomatics 86 Navigation, Banff, Canada, KIS1997, 289-299.

Verhagen S. (2004). Integer ambiguity validation: an open problem? GPS Solutions, 8, 36-43.

Verhagen S. (2005). The GNSS integer ambiguities: estimation and validation. Ph.D. thesis, Netherlands Geodetic Commission, Delft.

Verhagen S., Li B., Teunissen P.J.G., and Tiberius C.C.J.M. (2012). Challenges in ambiguity resolution: biases, weak models, and dimensional curse. Proceedings of 6th ESA Workshop on Satellite Navigation Technologies, NAVITEC 2012, 1-8.

Wang J., Lee H.K., Lee Y.J., Musa T., and Rizos C. (2005). Online stochastic modelling for network-based GPS real-time kinematic positioning. Journal of Global Positioning Systems, 4(1-2), 113-119.

Wanninger L. (1995). Enhancing differential GPS using regional ionospheric error models. Bulletin Géodésique, 69, 283-291.

Wanninger L. (2004). Ionospheric disturbance indices for RTK and Network RTK positioning. Proceedings of the 17th International Technical Meeting of the Satellite Division of the Institute of Navigation, ION GNSS 2004, 2849-2854. 
Wielgosz P., Grejner-Brzezinska D.A., and Kashani I. (2003). Regional ionosphere mapping with kriging and multiquadric methods. Journal of Global Positioning Systems, 2(1), 48-55.

Wielgosz P., Kashani I., and Grejner-Brzezinska D. (2005). Analysis of long-range network RTK during a severe ionospheric storm. Journal of Geodesy, 79, 524-531.

Received: 2013-12-19,

Reviewed: 2014-01-09, by S. Cellmer, Accepted: 2010-01-21. 